\title{
Complicaciones más frecuentes al inicio del uso de la CPAP en nuestro medio
}

\section{Most frequent complications at the beginning of the use of CPAP in our environment}

\author{
Emilio González García, MD, Phd. ${ }^{1}$ Rafael Valdivieso Martínez, MD, PhD. ${ }^{2}$
}

\section{Resumen}

INTRODUCCIÓN: al comienzo del tratamiento con presión positiva continua en la vía aérea (CPAP) por el síndrome de apnea/hipopnea del sueño (SAHS) pueden surgir distintos tipos de problemas que impidan su correcto uso o incluso su abandono. Por esto es preciso conocer cuáles son las principales complicaciones tanto para informar al paciente como para dar soluciones de forma temprana.

MATERIAL Y MÉTODO: se estudiaron a 120 pacientes diagnosticados con SAHS que comenzaron el tratamiento con CPAP nasal. Se realizaron cuestionarios para valorar su sueño previo al tratamiento e identificar los factores que influyeran en la adaptación a la CPAP. Pasados al menos 2 meses, se realizaron cuestionarios en los que preguntaba sobre las complicaciones surgidas y la tolerancia al tratamiento.

RESULTADOS: las molestias ocasionadas por la mascarilla o correas de fijación fueron las complicaciones más frecuentes, así como las lesiones ocasionadas por las mismas (callosidades, excoriaciones), que desaparecieron en 2-3 semanas. Es frecuente la sequedad de mucosas matutina y tiende a persistir. El ruido de los equipos causó molestias inicialmente, en especial a los acompañantes. Los episodios de congestión nasal y enrojecimiento ocular matutino se relacionaron con la mala fijación de la mascarilla. Un 38 \% no cumplió correctamente, con pocas horas de uso. 4 pacientes rechazaron el tratamiento.

DISCUSIÓN: la CPAP nasal conlleva complicaciones iniciales a detectar precozmente ya que, aunque la mayoría son problemas esporádicos o breves, persisten en algunos pacientes y pueden causar mal cumplimiento del tratamiento. Pasados 2 meses con complicaciones, se aconseja valorar el cambio de mascarillas o emplear humidificadores. Desde las unidades de sueño, es preciso advertir al paciente de estas complicaciones y su desaparición normal.

Palabras clave: CPAP nasal, SAHS, terapias respiratorias.
${ }^{1}$ Servicio de Neurofisiología Clínica. Hospital de Manises. Valencia, España. ${ }^{2}$ Servicio de Neurofisiología Clínica. Hospital La Fe de Valencia. Valencia, España.

Autor de correspondencia

Emilio González García. Servicio Neurofisiología Clínica. Hospital de Manises. Av. de la Generalitat Valenciana, 50. Manises (Valencia) 46940. Teléfono: 628079243. Correo electrónico: egongar@gmail.com. Recibido: 11/4/2018. Aceptado: 11/12/2018. 
RESULTS: The discomfort caused by the mask and/or fixation straps were the most frequent complications, as well as the injuries caused by them (callosities, excoriations) disappearing in 2-3 weeks. Dryness of morning mucous membranes is frequent and tends to persist. The noise of the equipment initially caused discomfort, especially to the companions. Episodes of nasal congestion and morning eye redness were associated with poor fastening of the mask. $38 \%$ did not complete correctly, with few hours of use. Four patients rejected the treatment.

DISCUSSION: Nasal CPAP involves initial complications to be detected early, because although most are sporadic or brief problems, they persist in some patients and can cause a poor treatment completion. After two months with complications, it is recommended assessing the change of masks or using humidifiers. From the sleep units, it is necessary to warn the patient of these complications and their normal disappearance.

Keywords: nasal continuous positive airway pressure, sleep apnea syndrome, respiratory therapies.

\section{Introducción}

El síndrome de apnea/hipopnea del sueño (SAHS) es el trastorno respiratorio más frecuente durante el sueño, afecta al $4 \%-6 \%$ de los varones y al $2 \%-4 \%$ de las mujeres en las edades medias de la vida, y es más frecuente conforme la edad es más avanzada. El SAHS se caracteriza por la aparición durante el sueño de episodios repetidos de obstrucción completa (apneas) o parcial (hipopneas) de la vía respiratoria superior (1).

Los pacientes con SAHS deben seguir unas medidas generales, como pérdida de peso, cese del hábito tabáquico y evitar el alcohol o el uso de medicación sedante en las horas previas al sueño. Estos factores favorecen o agravan la obstrucción respiratoria durante el sueño, y en los casos de SAHS leve, puede ser suficiente el control de estas medidas para que no se produzcan apneas durante el sueño (1). Algunos pacientes con anomalías anatómicas de vías aéreas superiores son susceptibles de empleo de dispositivos de avance mandibular (2) o incluso tratamientos quirúrgicos. Sin embargo, el tratamiento de elección una vez diagnosticado en SAHS sigue siendo la presión positiva continua sobre la vía aérea, empleando la CPAP (3).

Con la CPAP aplicada con mascarilla nasal se evita el colapso de la vía aérea durante el sueño y la aparición de eventos respiratorios (apneas, hipopneas, ronquidos, limitaciones de flujo aéreo, entre otros). Con esto se normaliza la estructura del sueño nocturno, por lo que desaparece la sintomatología derivada del SAHS (4).
La eficacia del tratamiento no solo depende del adecuado ajuste de la presión de aire de la CPAP, sino de la tolerancia del paciente al uso del dispositivo. Es bien sabido que el uso de la CPAP puede ocasionar toda una serie de molestias y efectos adversos que pueden concluir en la negativa del paciente a seguir el tratamiento (5). La mayoría de las complicaciones surge durante las primeras semanas o incluso meses del comienzo de su uso.

\section{Pacientes y método}

Para nuestro estudio se han valorado a 120 pacientes diagnosticados de SAHS en la Unidad de Sueño del Hospital Francesc de Borja de Gandía (Valencia, España). A todos ellos se les confirmó el diagnóstico mediante polisomnografía (PSG) nocturna hospitalaria siguiendo los criterios de Rechtschaffen y Kales (6), y mostraron un índice de apnea/hipopnea mayor de 10 eventos respiratorios por hora. Luego del diagnóstico se les recetó la CPAP, pautando una presión de aire provisional calculada mediante la fórmula de Miljeteig y Hoffstein (7), pendiente de titulación definitiva con una nueva PSG nocturna hospitalaria.

Los pacientes llenaron un cuestionario elaborado en nuestro servicio para conocer distintos parámetros en relación con su sueño que puedan modificarse luego del tratamiento. Este cuestionario se realiza de forma habitual en nuestro servicio y también recoge la información de los especialistas que han consultado hasta llegar al diagnóstico de SAHS. En la Tabla 1 se reflejan las cuestiones planteadas en el mismo. 
Tabla 1. Temas valorados en el cuestionario previo al tratamiento

\begin{tabular}{|l|}
\hline Principales problemas de sueño \\
\hline Médicos consultados \\
Intervención en la vía aérea superior \\
Medicaciones que alteren el sueño \\
Tratamiento por ansiedad o depresión \\
\hline Dificultad respiratoria nasal o respiración bucal \\
\hline Episodios de epistaxis \\
Cefaleas \\
\hline Antecedentes de conjuntivitis \\
\hline Infecciones de vías aéreas altas frecuentes \\
Alergias o hipersensibilidad a materiales \\
¿Piensa que duerme mal? \\
\hline
\end{tabular}

Pasados al menos dos meses del inicio del tratamiento se citaron a los pacientes para la PSG nocturna de ajuste de presión de la CPAP. En ese momento realizaron el segundo cuestionario, en el que se consultaron las posibles complicaciones aparecidas en el período inicial del tratamiento, así como su grado de cumplimiento y eficacia del mismo. En la Tabla 2 se muestran las cuestiones planteadas.

Tabla 2. Temas valorados en el cuestionario realizado luego de iniciar el tratamiento

\begin{tabular}{|l|}
\hline Tiempo de tratamiento con CPAP \\
Cumplimiento del tratamiento \\
Dificultad respiratoria nasal con la CPAP \\
Respiración bucal con la CPAP \\
Molestias causadas por mascarilla y correas \\
Lesiones por el uso de mascarilla y correas \\
Congestión nasal nocturna \\
Sequedad buconasal al despertar \\
Molestias por el ruido del equipo (paciente/acompañante) \\
Enrojecimiento ocular matutino \\
Cefalea matutina \\
Episodios de epistaxis \\
Frialdad del aire por la CPAP \\
Episodios de insomnio \\
Flatulencias o molestias gastrointestinales matutinas \\
¿Duerme mejor con la CPAP? \\
\hline
\end{tabular}

\section{Resultados}

De los 120 pacientes estudiados, 14 de ellos fueron mujeres y 106 hombres. La media de edad fue de 52 años, con una desviación estándar de 12 años, y edades que oscilan entre los 25 y los 80 años. Todos ellos presentaban un índice de apnea/hipopnea superior a 10 eventos por hora de sueño en la PSG diagnóstica, con una media de 57,3 y una desviación estándar de 25,9. Casi la totalidad de los pacientes presentaba un SAHS de grado moderado a muy grave; leve (índice de apneas/hipopneas [IAH]: 5-14,9); moderado (IAH: 15-29,9); grave $(\mathrm{IAH}>30)$; muy grave $(\mathrm{IAH}>50)(8)$.

La mayoría de los pacientes $(75,8 \%)$ ha consultado sobre sus problemas de sueño al médico de familia, y el resto fue remitido directamente desde otros especialistas. La especialidad más consultada fue neumología (66,7 \%), aunque el $80,8 \%$ ha consultado a más de un especialista antes de realizarse el diagnóstico (Figura 1).

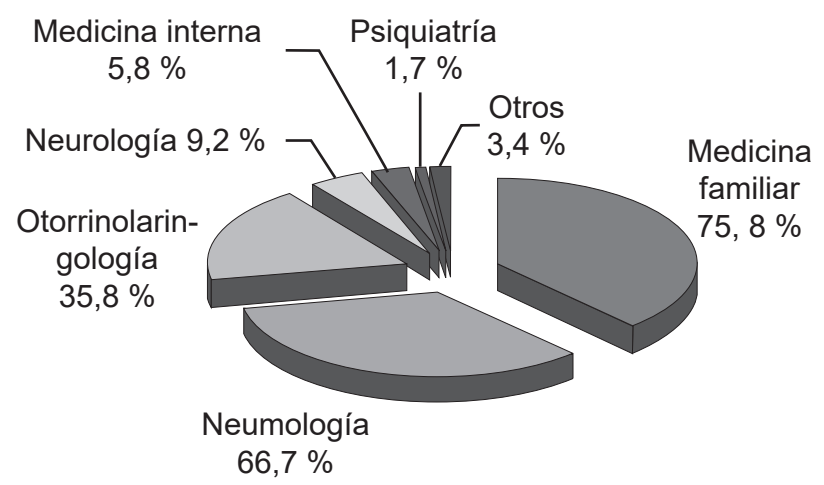

Figura 1. Porcentaje de pacientes que han visitado a cada especialidad médica hasta llegar al diagnóstico.

El problema consultado con más frecuencia es la presencia de ronquido durante el sueño (54,2\%), seguido de la hipersomnia diurna y la observación de apneas durante el sueño, con un $35 \%$ y un $33 \%$, respectivamente. Un $21,7 \%$ refirió sueño insuficiente o poco reparador, mientras un 10,8 \% consultó por otros problemas muy variados como disfunción eréctil, alteración de la memoria, hipertensión arterial mal controlada, entre otros. De todos ellos, un 18,3\% dijo no tener ningún problema, acudiendo a la consulta por 
indicación de la persona con la que comparte el sueño, siendo esta la que se queja de no poder dormir por culpa del paciente.

Unos 29 de los 120 pacientes han sido intervenidos en alguna ocasión por un otorrinolaringólogo, aunque solo 14 de los 120 han sido intervenidos en relación con su problema respiratorio nocturno.

El uso de medicación que puede alterar el sueño es frecuente en nuestro grupo de pacientes, que era de un $24,2 \%$ en el momento del estudio. Un $25,8 \%$ de los pacientes padecía o había llevado en algún momento tratamiento por ansiedad o depresión.

La postura preferida al dormir fue el decúbito lateral en $60 \%$, aunque un $22,5 \%$ muestra indiferencia al respecto. Duerme de forma habitual en decúbito supino un $9,2 \%$ y en decúbito prono un $8,3 \%$.

Los pacientes acudieron al ajuste de presión definitiva luego del período de adaptación superior a 4 semanas, con una media de 20 semanas desde la prescripción de la terapia ventilatoria. Solo un $45 \%$ refirió tolerar el equipo durante toda la noche; el resto no lo usaba durante toda la noche, con una media de 3-4 horas de uso. Un 20 \% reconoce no usarlo todos los días, con períodos variables de uso.

Un 48,3\% de los pacientes afirmaba no dormir bien antes. Una vez iniciado el tratamiento, el 43,3\% de los pacientes indicó dormir mejor con la CPAP. No mostró ningún cambio el 53,3\% de los pacientes e incluso un $4 \%$ indicó dormir peor.

Hasta un 59,1 \% reconoció tener dificultades respiratorias nasales (9) e incluso respiración bucal previamente, que persistió normalmente luego del tratamiento con la CPAP.

Las complicaciones más frecuentes fueron las molestias ocasionadas por la mascarilla y las correas de fijación (59,2\%), aunque en una media de 4 semanas y media (alrededor de un mes) desaparecieron. Solo un 7,5 \% siguió mostrando alguna molestia en el momento del ajuste de presión. En frecuencia, le sigue la sequedad buconasal al despertar, con un 49,2\% de los pacientes estudiados; un $20 \%$ seguía presentándola al momento del ajuste, al igual que los pacientes que lo sufrían de forma ocasional. 5 de los pacientes llevaban humidificador por esta causa.

El ruido ocasionado por los equipos también se muestra como un problema frecuente $(32,5 \%)$, aunque el 19,2\% de las quejas no fue del paciente, sino del acompañante. Tardaron en acostumbrase al mismo de 1 a 4 semanas y solo un acompañante se desplazó de la habitación.

Un 28,3 \% presentó algún tipo de lesión, irritaciones, excoriaciones, callosidades, entre otros, por el uso. En una media de 2,7 semanas ya no las presentaban. Ninguno mostraba lesiones pasado ese tiempo.

Un $25 \%$ refirió episodios de congestión nasal nocturna, casi todos ellos de forma ocasional, aunque 5 pacientes seguían mostrándolos en el momento del ajuste; 3 pacientes empezaron a usar humidificador $\mathrm{y}$ uno de ellos seguía el tratamiento con vasoconstrictores nasales (que ya usaba previamente).

Hasta un $20 \%$ se quejó de que el aire que salía de la CPAP era muy frío, un tercio de ellos solo de forma ocasional. 8 pacientes seguían quejándose en el momento del ajuste y 4 de ellos empezaron a usar humidificador/calentador del aire. Un 3,3 \% refirió no volver a notar la frialdad del aire entre 1 semana y 1 mes.

Al menos un $20 \%$ refirió algún episodio de insomnio, aunque solo 3 pacientes mostraban verdadera dificultad para mantener el sueño nocturno o despertares precoces. Un paciente dejó de usar la CPAP porque no podía conciliar el sueño.

Menos frecuente es la aparición de aerofagia (18,3 $\%$ ). La mitad seguía presentándola al inicio del ajuste y la otra mitad dejó de presentarla entre 1 semana y 1 mes del inicio del tratamiento.

El enrojecimiento ocular se pudo observar en un 17,5 $\%$ de los pacientes al principio del tratamiento. 7 pacientes $(5,8 \%)$ seguían mostrándolo en el momento del ajuste, el resto solo llegó a sufrirlo de forma ocasional o cedió. 1 paciente ya sufría de conjuntivitis crónica. 
Solo 10 pacientes aquejaron algún tipo de cefalea matutina, aunque en 3 de ellos era previa al tratamiento; $\mathrm{y}$ el resto al inicio o de forma ocasional.

Episodios de epistaxis solo se registraron en un paciente en tratamiento con anticoagulantes orales (Sintrom $^{\circledR}$ ). 5 pacientes presentaron algún tipo de sangrado poco relevante, al retirase las secreciones nasales; dicho suceso no duró más de 1 semana.

La casi totalidad de los pacientes $(92,5 \%)$ usó para la adaptación una mascarilla nasal Flexifit $^{\circ}$ de Fisher \& Paykel. Otras mascarillas nasales de Resmed y Respironics se usaron con el resto. 8 pacientes acudieron usando mascarillas nasobucales y 1 usó mascarilla de olivas nasales.

La presión media de ajuste de estos pacientes fue 9 $\mathrm{cm}$ de agua, con una desviación estándar de $\pm 2,14 \mathrm{~cm}$ de agua y presiones entre 4 y $14 \mathrm{~cm}$ de agua.

Hasta 30 pacientes presentaron problemas de adaptación durante la titulación definitiva de presión, mostrando en su mayoría fugas por mala colocación y su- jeción de la mascarilla, y respiración bucal que podía controlarse con mentonera para evitar la apertura bucal durante el sueño. Luego del ajuste se precisó un cambio de mascarilla nasal a nasobucal por la incapacidad para evitar la respiración bucal en 13 pacientes. Solo en 1 paciente no se pudo terminar el estudio, se negó a continuar con la prueba por la imposibilidad para conciliar el sueño.

Posteriormente, de los 120 pacientes solo 4 solicitaron el cese del tratamiento. Todos ellos mostraron alguna complicación que no consiguió solucionarse pese a los cambios de mascarilla. La mayoría mostraba imposibilidad para la respiración nasal e imposibilidad para conciliar el sueño con la CPAP.

\section{Discusión}

Comenzar a usar una CPAP implica una serie de molestias que pueden llevar no solo a un mal cumplimiento del tratamiento sino a la negación del paciente a seguirlo. Es muy frecuente la aparición de estas complicaciones (Figura 2), pero son fáciles de identificar; su duración y persistencia son poco frecuentes (Tabla 3).

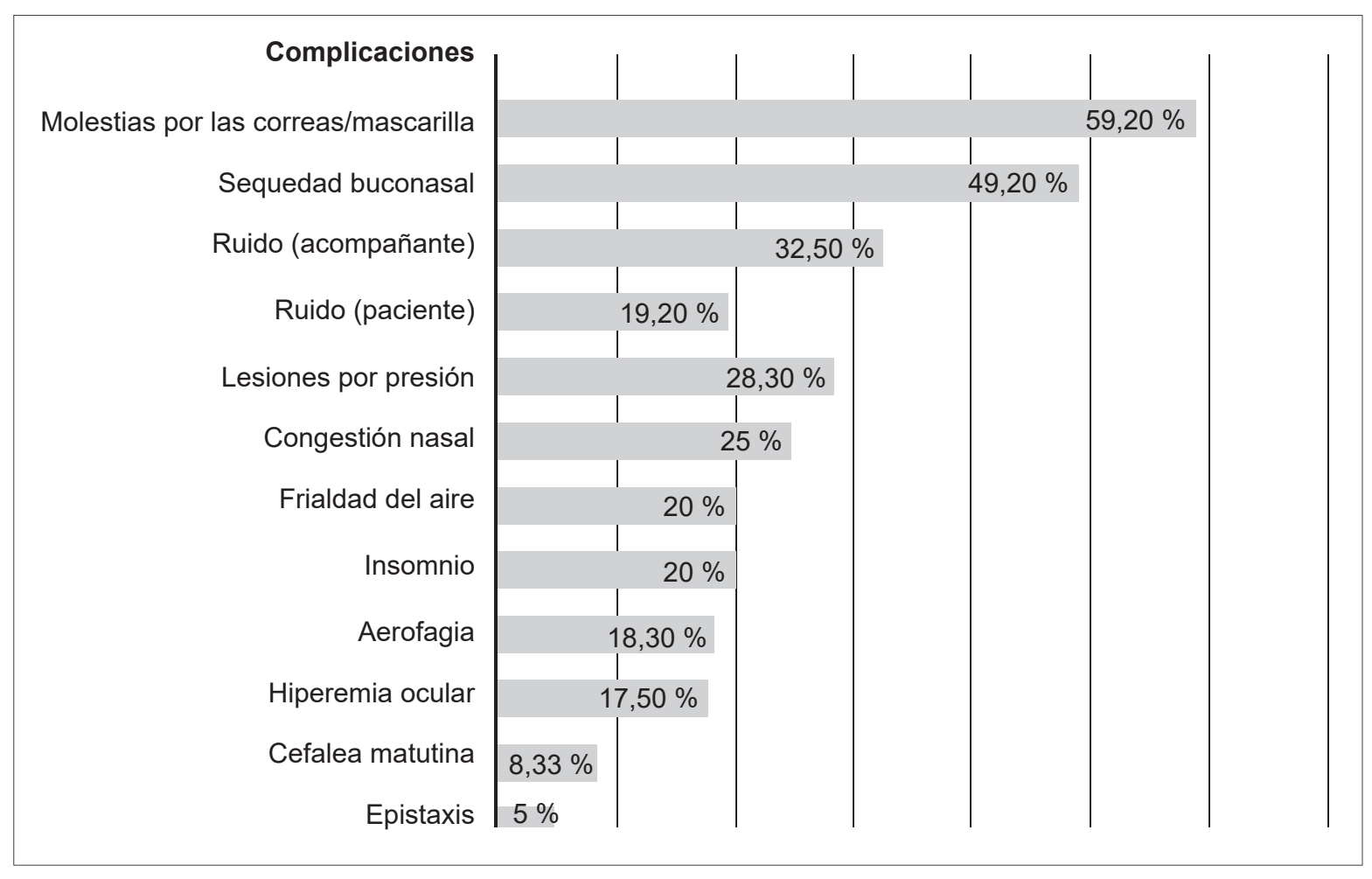

Figura 2. Porcentaje de pacientes que han sufrido complicaciones con el uso de la CPAP. 
Tabla 3. Complicaciones del uso de la CPAP

\begin{tabular}{|lll|}
\hline \multicolumn{1}{c}{ Complicaciones } & \multicolumn{1}{c|}{ Duración media } & Problema persistente \\
\hline Molestias por las correas/mascarilla & 4,5 semanas & $7,5 \%$ \\
\hline Sequedad buconasal & ocasional & $20 \%$ \\
\hline Ruido & 2,75 semanas & $<1 \%$ \\
\hline Lesiones por presión & 2,75 semanas & -- \\
\hline Congestión nasal & Ocasional & $4,17 \%$ \\
\hline Frialdad del aire & Ocasional & $6,7 \%$ \\
\hline Insomnio & Ocasional & $<1 \%$ \\
Aerofagia & Ocasional & $9,2 \%$ \\
\hline Hiperemia ocular & Ocasional & $5,8 \%$ \\
Cefalea matutina & Ocasional & $<1 \%$ \\
Epistaxis & Ocasional & $<1 \%$ \\
\hline
\end{tabular}

Más de la mitad de los pacientes se queja de molestias por mascarilla o correas de fijación; sin embargo, en cuestión de 1 mes desaparecen. Solo unos pocos $(7,5 \%)$ dicen que todavía les molesta, pero de forma mínima y no les dificulta su uso.

Casi la mitad refiere sequedad buconasal, aunque la mayoría de ellos solo de forma ocasional. En este caso, el problema suele ser persistente, pero en el momento del ajuste se observa respiración bucal en muchos de ellos. En caso de mantenerse el problema, es conveniente plantearse el uso de humidificadores del aire.

A un tercio de las consultas les molestó el ruido del mecanismo ventilatorio, aunque menos de la mitad lo aquejó el paciente, pero los acompañantes fueron los más afectados. En cualquier caso, en menos de 1 mes se adaptó al ruido casi la totalidad, por lo que en muy raras ocasiones debe plantearse el cambio de equipo.

La presión de la mascarilla y las correas puede ocasionar lesiones sobre la piel, excoriaciones, irritación, callosidades, entre otros. Aunque es algo frecuente, entre 2 y 3 semanas de uso continuado ya no se observan. Algunos pacientes dejan de ponerse la mascarilla o la usan pocas horas, por lo que no dejan tiempo a que la piel de la cara se adapte a la presión de los materiales. En pieles muy sensibles o en pacientes con alteraciones anatómicas faciales complejas, es posible cambiar de mascarilla a olivas nasales o interfaces de silicona.
Un cuarto de los pacientes presenta episodios ocasionales de congestión nasal nocturna, solo persistentes en muy pocos pacientes $(4,17 \%)$. En esos casos sería precisa la valoración por el otorrinolaringólogo o el cambio a mascarilla buconasal.

Hasta un quinto de los pacientes dice notar el aire de la mascarilla muy frío, pero solo se mantiene el problema en pocos de ellos, a los que se les puede recetar un calentador-humidificador del aire o simplemente un buen acondicionamiento de la habitación.

Los pacientes no llegan a sufrir insomnio propiamente dicho, tan solo algunas noches les es muy difícil llegar a conciliar el sueño o muestran despertares precoces. Que ello se convierta en un problema de sueño es muy poco frecuente y solo lo hemos visto en pacientes que han sufrido insomnio previo al estudio.

Algunos pacientes $(8,3 \%)$ se quejan de molestias gastrointestinales y gases durante la mañana. Es un problema que suele persistir; sin embargo, estos pacientes suelen manifestar respiración bucal. Adaptarse a la respiración nasal suele poner fin a estos problemas.

Hasta un $17,5 \%$ presenta hiperemia ocular al despertar. Si este problema se mantiene, pueden producirse infecciones oculares (10) u otras complicaciones a ese nivel. Con la correcta adaptación de la mascarilla se evitan las fugas de aire, principales causantes de es- 
tas complicaciones. Una mala higiene de la mascarilla también se asocia con este problema.

Son poco frecuentes y ocasionales los episodios de cefalea matutina, que ceden en poco tiempo con el uso de la CPAP. Suele darse en pacientes que ya las sufrían previamente.

La complicación menos frecuente es la epistaxis (11). Lo normal es observar solo pequeños sangrados al retirar las secreciones nasales por la mañana, debido a la sequedad de las mucosas. Si el problema persiste, pueden ser tratados con humidificadores.

Más de la mitad de los pacientes no usó la CPAP de forma correcta hasta el ajuste de presión. Los pacientes que mejor completaron el tratamiento fueron aquellos con SAHS más grave (12).

Hay que tener en cuenta que hasta un $80,8 \%$ de los pacientes ha consultado a más de un especialista médico antes de llegar al diagnóstico y tratamiento de su SAHS. Por esto es necesaria la organización de las Unidades de Sueño, en las que un equipo multidisciplinar atiende a pacientes con la sospecha de SAHS, evitando el retraso del tratamiento que supone ser remitido de una consulta a otra. Se debe tener especial atención en pacientes roncadores (el problema más consultado) y entrevistar a los pacientes con su acompañante nocturno, ya que es frecuente que solo esa persona sea consciente de que el paciente tenga un problema de sueño.

\section{Conclusión}

La aparición de complicaciones al inicio del tratamiento con CPAP es muy común. Las más frecuentes resultan ser las molestias ocasionadas por los mecanismos de fijación o la propia mascarilla; sin embargo, son las primeras en desaparecer de forma espontánea solamente con el uso continuado de la CPAP.

Es preciso advertir a los pacientes de todas estas posibles complicaciones ya que la mayoría de ellas desaparece en las primeras semanas si se usa la mascarilla de forma correcta todas las noches durante el tiempo requerido. Por esto es necesaria la existencia de consultas tanto médicas como de enfermería para explicar el funcionamiento y las molestias que puede producir la CPAP. Con ello se asegura el correcto uso del equipo y de la adaptación al mismo, evitando que los pacientes dejen el tratamiento.

Con una consulta a los 2 meses del comienzo del tratamiento, se identifican aquellas complicaciones que no han desaparecido por completo, pudiendo entonces ponerles solución, ya sea con mecanismos humidificadores-calentadores del aire o con el cambio de mascarillas.

\section{Referencias}

1. Grupo Español de Sueño (GES). Consenso Nacional sobre el síndrome de apneas-Hipopneas del sueño (SAHS). Arch Bronconeumol. 2005;41(54):7-9.

2. Cistulli PA, Gotsopoulos H, Marklund M, Lowe AA. Treatment of snoring and obstructive sleep apnea with mandibular repositioning appliances. Sleep Med Rev. 2004;8(6):443-57.

3. Marín JM, Kimoff JM, Cosío MG. Síndrome de apnea obstructiva del sueño y su tratamiento mediante presión positiva aérea continua. Arch Bronconeumol. 1990;26(6):255-61.

4. Márquez-Báez C, Paniagua-Soto J, Castilla-Garrido JM. Treatment of sleep apnea syndrome with CPAP: compliance with treatment, its efficacy and secondary effects. Rev Neurol. 1998;26(151):375-80.

5. Hoffstein V, Viner S, Mateika S, Conway J. Treatment of obstructive sleep apnea with nasal continous positive airway pressure. Patient compliance, percepcion benefits and side effects. Am Rev Respir Dis. 1992;145(4 Pt 1):841-5.

6. Rechtschaffen A, Kales A. A manual for standardized terminology, techniques and scoring system for sleep stages of human subjects. Washington, D. C.: Public Health Service, U. S. Government Printing Office; 1968.

7. Miljeteig H, Hoffstein V. Determinants of continuous positive airway pressure level for treatment of obstructive sleep apnea. Am J Respir Crit Care Med. 1993;147(6 Pt 1):1526-30.

8. McNicholas WT. Diagnosis of obstructive sleep apnea in adults. Proc Am Thorac Soc. 2008;5(2):154-60.

9. Esteller E, Matiñó E, Segarra F, Sanz JJ, Ademà JM, Estivill E. Efectos adversos derivados del tratamiento con nCPAP y su relación con la nariz. Acta Otorrinolaringol Esp. 2004;55(1):17-22.

10. Harrison W, Pence N, Kovacich S. Anterior segment complications secondary to continuous positive airway pressure machine treatment in patients with obstructive sleep apnea. Optometry. 2007;78(7):352-55.

11. Strumpf DA, Harrop P, Dobbin J, Millman RP. Massive epistaxis from nasal CPAP therapy. Chest. 1989;95(5):1141.

12. Yetkin O, Kunter E, Gunen H. CPAP compliance in patients with obstructive sleep apnea syndrome. Sleep Breath 2008;12(4):365-7. 